\title{
Effect of respiration on the characteristic ratios of oscillometric pulse amplitude envelope in blood pressure measurement
}

\author{
Yihan Gui, Fei Chen Member, IEEE, Alan Murray, Dingchang Zheng
}

\begin{abstract}
Systolic and diastolic blood pressures (BPs) are the most important physiological parameters for disease diagnosis. Systolic and diastolic characteristic ratios derived from oscillometric pulse waveform have been widely used to estimate automated non-invasive BPs in oscillometric BP measurement devices. The oscillometric pulse waveform is easily influenced by respiration, which may cause variability to the characteristic ratios and subsequently BP measurement. This study quantitatively investigated how respiration patterns (i.e., normal breathing and deep breathing) affect the systolic and diastolic characteristic ratios. The study was performed with clinical data collected from 39 healthy subjects, and each subject conducted BP measurements during normal and deep breathings. Analytical results showed that the systolic characteristic ratio increased significantly from $0.52 \pm 0.13$ under normal breathing to $0.58 \pm 0.14$ under deep breathing $(p<0.05)$, and the diastolic characteristic ratio was from $0.75 \pm 0.12$ under normal breathing to $0.76 \pm 0.13$ under deep breathing $(p>0.05)$. In conclusion, deep breathing significantly affected the systolic characteristic ratio, suggesting that automated oscillometric BP device which is validated under resting condition should be strictly used for measurements under resting condition.
\end{abstract}

\section{INTRODUCTION}

Automated non-invasive blood pressure (BP) measurement devices are widely used in clinical measurements, and the majority of them are based on the oscillometric technique. The oscillometric non-invasive BP devices estimate systolic blood pressures (SBP) and diastolic blood pressures (DBP) by analyzing the pressure pulse variation induced in a pressurized cuff around the limb $[1,2]$. Nowadays, the oscillometric waveform is often analyzed by using empirical algorithms to determine BPs. As the cuff pressure is reduced from above the SBP to below the DBP, the peak of the oscillometric pulse first increases as the cuff pressure decreases from above the SBP to the mean arterial pressure (MAP), after which it decreases when the cuff

This study was supported by the National Natural Science Foundation of China (Grant No. 61571213). It was also supported by the Engineering and Physical Sciences Research Council (EPSRC) Healthcare Partnership Award (reference number EP/I027270/1), and EPSRC standard grant (reference number EP/F012764/1).

Yihan Gui is with the Department of Electrical and Electronic Engineering, Southern University of Science and Technology, Shenzhen, China (e-mail: guiyh@mail.sustc.edu.cn).

Fei Chen is with Department of Electrical and Electronic Engineering, Southern University of Science and Technology, Shenzhen, China (corresponding author to provide phone: (86) 755-8801-8554; e-mail: fchen@sustc.edu.cn).

Alan Murray is with School of Electrical and Electronic Engineering and Faculty of Medical Sciences, Newcastle University, Newcastle upon Tyne, UK (e-mail: alan.murray@newcastle.ac.uk).

Dingchang Zheng is with Health and Wellbeing Academy, Anglia Ruskin University, Chelmsford, UK (e-mail: dingchang.zheng@anglia.ac.uk).

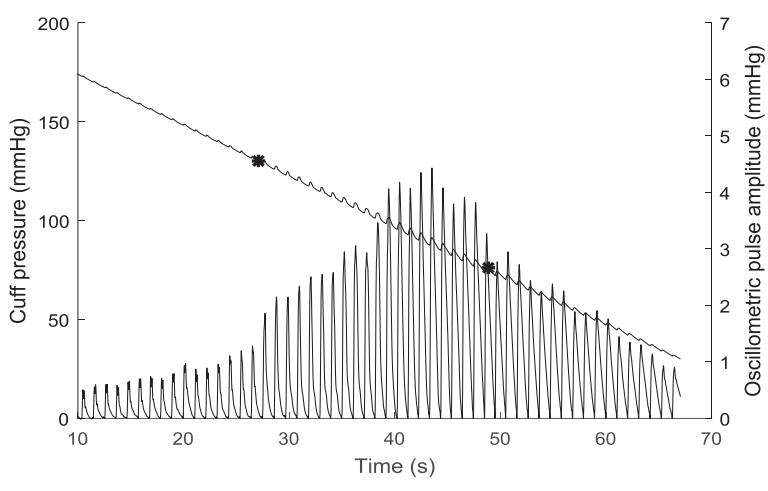

Figure 1. Deflating cuff pressure and the extracted oscillometric pulses as the cuff pressure is decreased from 170 to $30 \mathrm{mmHg}$. The two stars on the cuff pressure indicate the manual auscultatory systolic (left) and diastolic (right) blood pressures $(130 / 76 \mathrm{mmHg})$ measured for this subject.

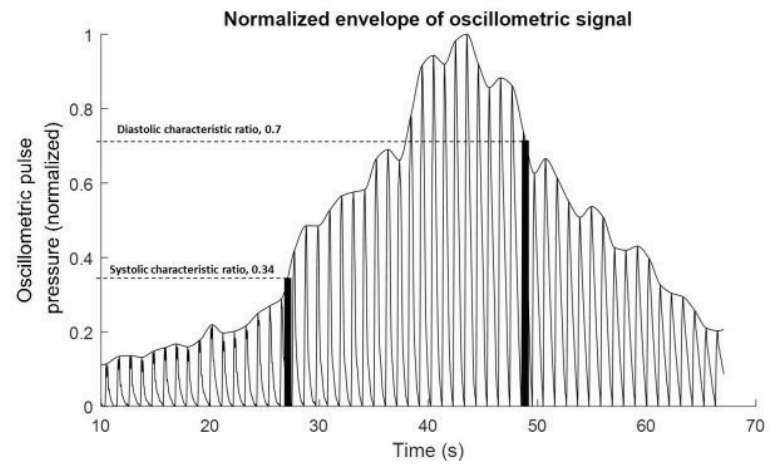

Figure 2. The oscillometric pulses in Fig. 1 and the oscillometric pulse envelope, which are normalized to the maximum peak amplitude of the oscillometric pulses. The solid vertical lines represent the timing positions of systolic (left) and diastolic (right) characteristic ratios, respectively. The dashed horizontal lines denote the values of systolic (low) and diastolic (top) characteristic ratios.

pressure further decreases below the DBP (see example in Fig. 1) [5].

Many studies have attempted to investigate the relationship between the features of oscillometric waveform and the systolic and diastolic BPs, and defined a series of indices including the systolic and diastolic characteristic ratios [2]. As illustrated in Fig. 2, with auscultatory BPs as reference, the systolic and diastolic characteristic ratios can be calculated from the oscillometric waveform by dividing the amplitudes of oscillometric pulses at the manual auscultatory systolic and diastolic pressures, respectively, by the maximum pulse peak amplitude, as: 
$\underline{\text { Systolic characteristic ratio }}=$ Amplitude in the envelope of oscillometric pulse where the cuff pressure equals the auscultatory systolic pressure / peak amplitude of the maximum oscillometric pulse

Diastolic characteristic ratio $=$ Amplitude in the envelope of oscillometric pulse where the cuff pressure equals the auscultatory diastolic pressure / peak amplitude of the maximum oscillometric pulse

Systolic and diastolic characteristic ratios have been widely used in non-invasive BP measurement devices for automated BP determinations [2, 4]. Earlier studies have shown that the systolic and diastolic characteristic ratios varied with different conditions, and among different populations [2-4]. Geddes et al. [2] reported a systolic characteristic ratio range from 0.45 to 0.57 and a diastolic characteristic ratio range from 0.69 to 0.89 for healthy subjects under resting condition. Other studies noted that the ratios varied with arterial wall viscoelastic properties and arterial pressure pulse amplitudes [4], and indicated that the typical systolic and diastolic characteristic ratios varied from 0.46 to 0.64 and from 0.59 to 0.80 , respectively [4], similar to the results reported by Geddes et al [2]. Hence, due to the variability of characteristic ratios, using fixed characteristic ratios for BP determination may potentially cause BP measurement bias or error.

Our previously published work has reported the modulation effect of respiration on oscillometric pulses [6,7]. Thus, it is reasonable to hypothesize that deep breathing may affect the values of characteristic ratios. However, it has not been quantified to what extent the deep breathing affects the variability of both systolic and diastolic characteristic ratios during BP measurement. Therefore, the aim of this study was to quantitatively investigate the effect of breathing on the systolic and diastolic characteristic ratios.

\section{METHOD}

The study recruited 39 healthy participants ( 26 males and 13 females; aged from 23 to 65 years) according to the Declaration of Helsinki (1989) of the World Medical Association. Their mean \pm SD of age, height and weight were $39 \pm 16$ years, $175 \pm 8 \mathrm{~cm}$ and $72 \pm 10 \mathrm{~kg}$, respectively. This study was approved by Newcastle and North Tyneside NHS Research Ethics Committee, and informed and written consent was obtained from all participants.

For each participant, manual auscultatory SBP and DBP were measured by a trained operator following the BP measurement guidelines of the British and European Hypertension Societies [8] under both resting and deep breathing conditions. The cuff pressure was inflated to 200 $\mathrm{mmHg}$, and then deflated linearly at a recommended rate of $2-3 \mathrm{mmHg} / \mathrm{s}$. Two cuff pressure signals (from resting and deep breathing conditions) were digitally recorded at a sampling rate of $2 \mathrm{kHz}$.

Oscillometric pulses were then derived from the cuff pressure signal. The signal processing procedure to obtain characteristic ratios included three steps: (1) extracting the oscillometric pulse signal from the originally recorded cuff pressure signal, as shown in Figs. 3 (a) and (b); (2) forming the envelope of the oscillometric pulse signal using cubic

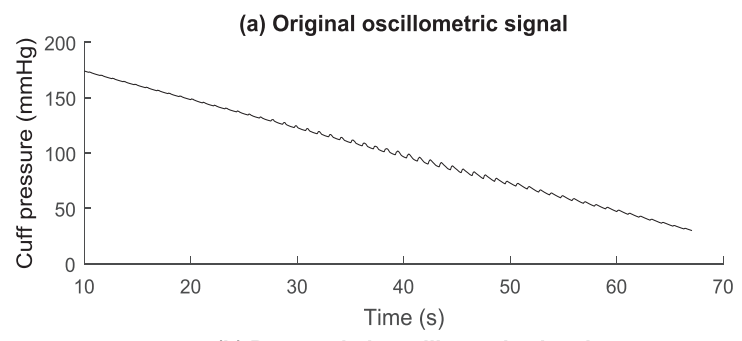

(b) Dentrended oscillometric signal

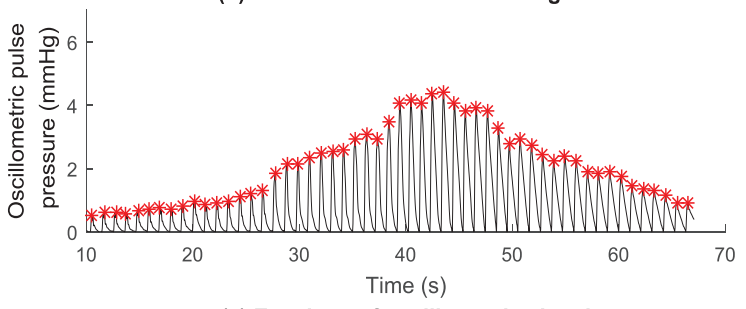

(c) Envelope of oscillometric signal
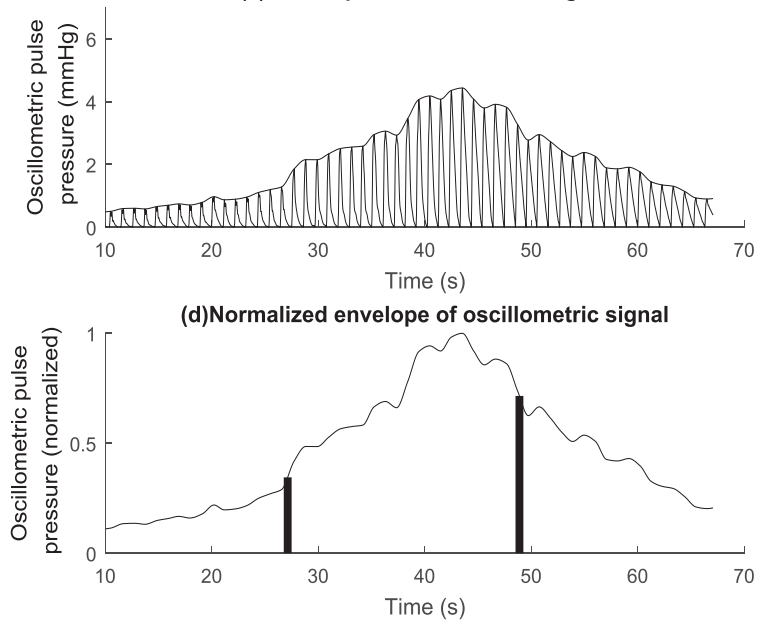

Figure 3. The procedure to extract oscillometric pulse envelope from deflating cuff pressure. Panel (a) shows that as the cuff pressure deflates, the original cuff pressure signal is reduced from 175 to 30 $\mathrm{mmHg}$. Panel (b) shows oscillometric pulses after removing the baseline cuff pressure (the linear component of cuff pressure is removed). Panel (c) shows the envelope of oscillometric pulses by a cubic interpolation. Panel (d) shows the normalized envelope and the positions (solid vertical lines) of the systolic (left) and diastolic (right) characteristic ratios referred to the manual auscultatory BPs.

interpolation as showed in Fig. 3 (c); and (3) using the manual auscultatory BP measurements to obtain the systolic and diastolic characteristic ratios according to Equ. (1) and Equ. (2). More details on implementing each signal processing step are described below. Analysis was performed on anonymized data.

\section{A. Extracting oscillometric pulses from cuff pressure signal}

Figure 3 (a) illustrates the procedure of extracting oscillometric pulses from the originally recorded cuff pressure signal. The cuff pressure signal showed in Fig. 3 (a) was acquired from one subject during BP measurement as the cuff pressure was reduced from 175 to $30 \mathrm{mmHg}$, and low-pass filtered with cutoff frequency of $10 \mathrm{~Hz}$. In this de-trending processing, the linear component of the cuff pressure signal, in other words, the linearly deflated baseline cuff pressure was removed. 


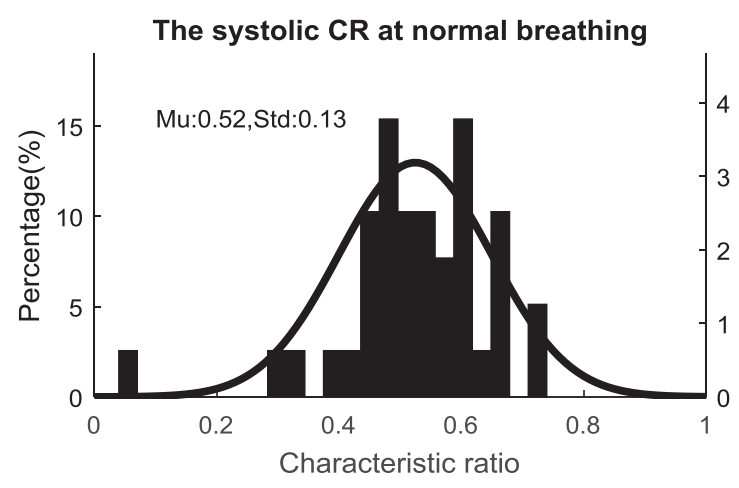

(a)

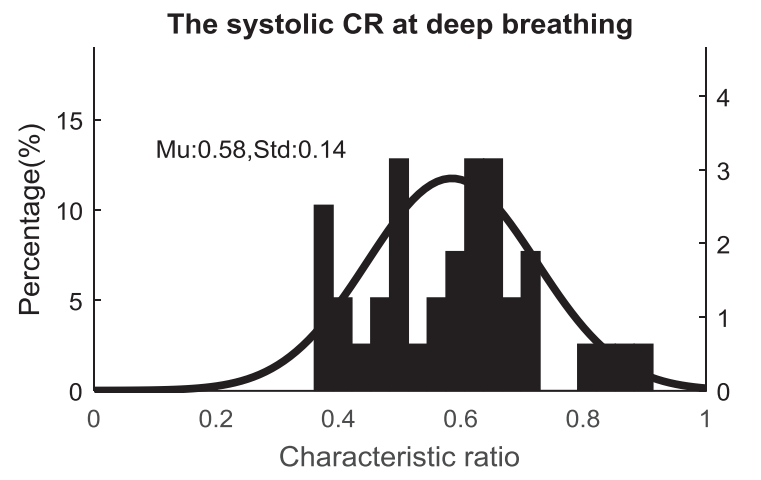

(c)

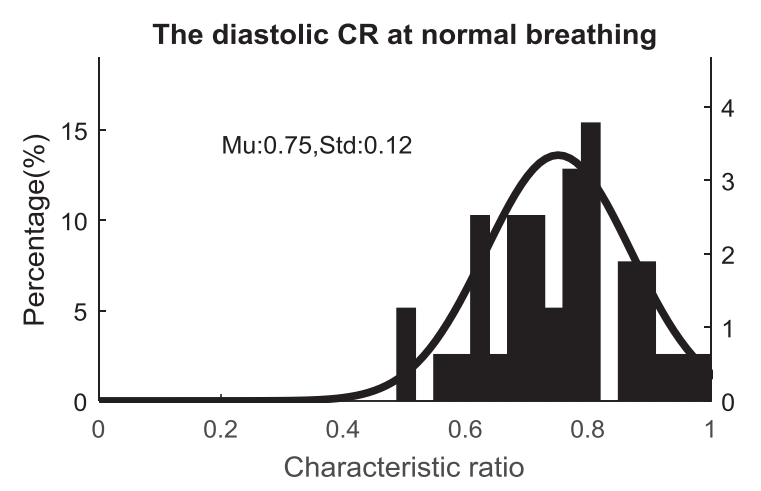

(b)

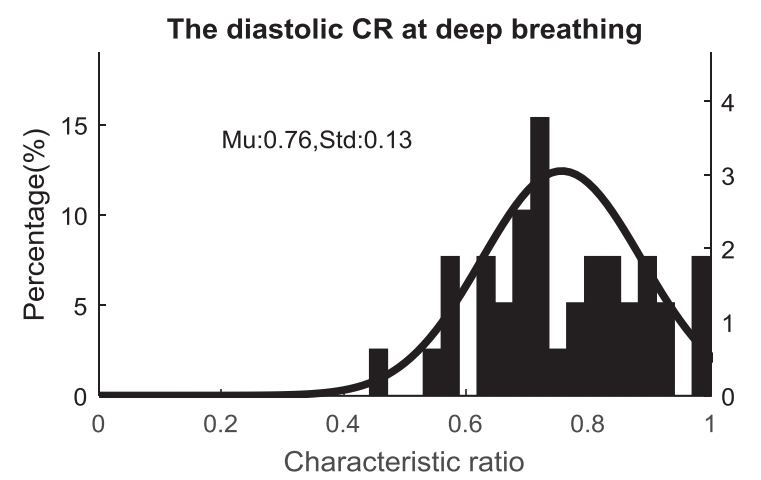

(d)

Figure 4. Histograms and fitted normal distributions of systolic and diastolic characteristic ratios (CRs) for both the normal and deep breathing conditions. (Mu: the mean of characteristic ratios; Std: the standard deviation of characteristic ratios).

\section{B. Interpolating oscillometric pulse peaks to form envelope}

The peaks of all oscillometric pulses were identified first, and marked in red in Fig. 3 (b). The envelope of the oscillometric signal was then constructed through cubic interpolation between oscillometric pulses.

\section{Calculating systolic and diastolic characteristic ratios}

Given the manual auscultatory SBP and DBP, the corresponding systolic and diastolic measuring points in the cuff pressure waveform and oscillometric pulse signal were located, as shown in Fig. 1, where the characteristic ratios were derived according to Equ. (1) and Equ. (2). As shown in Fig. 3 (d), the left thick line indicates the position of systolic characteristic ratio and the right line for the diastolic characteristic ratio.

\section{RESULT}

Figure 4 shows that the systolic characteristic ratio increased significantly from $0.52 \pm 0.13$ under normal breathing to $0.58 \pm 0.14$ under deep breathing $(p<0.05)$, and the diastolic characteristic ratio was from $0.75 \pm 0.12$ under normal breathing to $0.76 \pm 0.13$ under deep breathing $(p>0.05)$.

Specifically, under normal breathing, the systolic and diastolic characteristic ratios varied from 0.04 to 0.74 and from 0.49 to 0.99 , respectively. $69 \%$ of the systolic characteristic ratios were within the range $0.3-0.6$, whereas $82 \%$ of the diastolic characteristic ratios were between 0.6 and
0.9. Four waveforms had diastolic characteristic ratios greater than 0.9 , and two had ratios greater than 0.95 .

Under deep breathing, the systolic and diastolic characteristic ratios varied from 0.36 to 0.91 and from 0.44 to 0.99, respectively (Fig. 4). 51\% of the systolic characteristic ratios were within the range $0.3-0.6$, whereas $66 \%$ of the diastolic characteristic ratios were between 0.6 and 0.9. Eight waveforms had diastolic characteristic ratios greater than 0.9 , and three had ratios greater than 0.95 .

\section{CONCLUSION}

The present work investigated how the systolic and diastolic characteristic ratios changed with deep breathing in comparison with normal resting condition. A significant effect of breathing on systolic characteristic ratio has been demonstrated during BP measurement, i.e., from 0.52 under normal breathing to 0.58 under deep breathing. The change of characteristic ratio caused by respiration may subsequently cause BP measurement error from automated oscillometric BP measurement. In other words, the value of characteristic ratio needs to reflect the influence of respiratory modulation. Hence, automated oscillometric BP device which is validated under resting condition should be strictly used for measurements under resting condition, and the intelligent adaptive adjustment of characteristic ratios (particularly systolic characteristic ratio) could be considered in response to respiration pattern in the future when designing oscillometric pulse based automated BP devices. 


\section{REFERENCES}

[1] Maynard Ramsey III, "Noninvasive automatic determination of mean arterial blood pressure," Med Biol Eng Computing 1979; 17: 11-18.

[2] L. A. Geddes, M. Voelz, C. Combs, D. Reiner and C. F. Babbs,

"Characterization of the oscillometric method for measuring indirect blood pressure," Annal Biomed Eng 1982; 10: 271-280.

[3] H. Groß, S. Mieke and M. Ulbrich, "An arm phantom: a digital simulation system for testing sphygmomanometers," Med Eng \& Tech 1996; 20: 75-83.

[4] M. Ursino and C. Cristalli, "A mathematical study of some biomechanical factors affecting the oscillometric blood pressure measurement," IEEE Trans Biomed Eng 1996; 43: 761-778.

[5] J. N. Amoore, E. Vacher, I. C. Murray, S. Mieke, S. T. King, F. E. Smith and A. Murray "Effect of the shapes of the oscillometric pulse amplitude envelopes and their characteristic ratios on the differences between auscultatory and oscillometric blood pressure measurements," Blood Pressure Monitoring 2007: 297-305.

[6] D. Chen, F. Chen, A. Murray and D. Zheng, "A method for extracting respiratory frequency during blood pressure measurement, from oscillometric cuff pressure pulses and Korotkoff sounds recorded during the measurement," Proc IEEE Eng Med Biol Soc, 2016: 4268.

[7] D. Chen, F. Chen, A. Murray and D. Zheng, "Respiratory modulation of oscillometric cuff pressure pulses and Korotkoff sounds during clinical blood pressure measurement in healthy adults," BioMedical Engineering OnLine 2016: 53.

[8] E. O’Brien, R. Asmar, L. Beilin, Y. Imai, J.M. Mallion, G. Mancia et al., "European Society of Hypertension recommendations for conventional, ambulatory and home blood pressure measurement," J Hypertens, 2003; 21: $821-848$ 